\title{
PRODUTIVIDADE DE RAÍZES DE MANDIOCA EM FUNÇÃO DE ÉPOCAS E NÍVEIS DE DESFOLHA
}

Amarílis Beraldo Rós, Andréia Cristina Silva Hirata

Agência Paulista de Tecnologia dos Agronegócios, Polo Regional da Alta Sorocabana, Presidente Prudente, SP. E-mail: amarilis@apta.sp.gov.br.

\section{RESUMO}

Nesse trabalho teve-se por objetivo avaliar a produtividade da cultura da mandioca submetida a diferentes épocas e níveis de desfolha. O trabalho foi realizado em Presidente Prudente/SP. O delineamento experimental foi em blocos ao acaso, com quatro repetições. Os tratamentos foram: sem desfolha (testemunha), desfolha de 25, 50 e 75\% em novembro de 2013 e desfolha de 25,50 e $75 \%$ no mês de fevereiro de 2014. O plantio foi realizado em junho de 2013 . Foi verificado que não houve diferença estatística na produtividade $\left(32 \mathrm{t} \mathrm{ha}^{-1}\right)$, números total (134.555 unidades $\mathrm{ha}^{-1}$ ) e comercial de raízes (89930 unidades ha ${ }^{-1}$ ), massa fresca individual $(374,8 \mathrm{~g})$, diâmetro (5 $\mathrm{cm}$ ) e comprimento de raízes $(26,5 \mathrm{~cm})$. A semelhança de valores entre os tratamentos pode estar relacionada a condições adequadas de solo e clima, o resultou em rápida recuperação e manutenção de elevada produtividade.

Palavras Chave: Manihot esculenta; desfolha artificial; formato de raiz; número de raiz.

\section{CASSAVA YIELD IN FUNCTION OF LEVEL AND TIME DEFOLIATION}

\section{ABSTRACT}

This study aimed to evaluate the cassava crop yield submitted to different levels and times defoliation. The work was conducted at Presidente Prudente city / São Paulo State. The experimental design was in randomized blocks with four replications. The treatments were: without defoliation (control), defoliation of 25, 50 and 75\% in November 2013 and defoliation of 25, 50 and $75 \%$ in February 2014. The planting was carried out in June 2013. It was verified that there was no statistical difference in yield $\left(32 \mathrm{t} \mathrm{ha}^{-1}\right)$, total $\left(134,555\right.$ units ha $\left.{ }^{-1}\right)$ and commercial roots numbers ( 89930 units $\mathrm{ha}^{-1}$ ), individual fresh mass $(374.8 \mathrm{~g})$, diameter $(5 \mathrm{~cm})$ and root length $(26.5 \mathrm{~cm})$. Similarity of values among the treatments could be related to adequate soil and climate conditions, which caused the plants to present a rapid recovery and to maintain high tield.

Keywords: Manihot esculenta; artificial defolation; root format; root number.

\section{INTRODUÇÃO}

A mandioca é uma planta originária da América Latina, sendo encontrada na forma nativa em vários países, inclusive o Brasil (ALLEM, 1994). Suas raízes ocupam o quinto lugar entre os alimentos mais produzidos no mundo, ficando atrás apenas do arroz, trigo, batata e milho (INTERNATIONAL POTATO CENTER, 2010). É cultivada em todos os estados brasileiros (IBGE, 2015), destacando-se na alimentação humana e animal e como matéria prima em ampla diversidade de produtos industriais.

A cultura apresenta elevada produtividade por unidade de área, sendo que a cultivar IAC 576-70, que é uma variedade de mesa, apresenta potencial de produzir mais de $40 \mathrm{t} \mathrm{ha}{ }^{-1}$, em um ciclo de oito meses (RÓS et al, 2013). No entanto, sua produtividade pode ser reduzida quando há incidência de pragas e /ou doenças na cultura. 
Uma das principais pragas da cultura é o mandarová (Erinnyis ello L.), lagarta que apresenta elevada capacidade de desfolha das plantas, reduzindo a área fotossinteticamente ativa das plantas. Ataques severos causam desfolha completa da planta, menor produtividade e qualidade de raiz (BELLOTTI et al., 1992). Segundo Bellotti et al. (1999), pode ocorrer a redução na produção de raízes, na ordem de 0 a $45 \%$ com um só ataque e até $64 \%$ com dois ataques, podendo variar em função da variedade, idade das plantas, fertilidade do solo, condições ambientais. O período mais crítico de seu ataque ocorre durante os cinco primeiros meses da cultura (LEONEL et al., 2015). A praga pode ocorrer durante todo o ano (SCHMITT, 2002). No Estado de São Paulo, a ocorrência do mandarová é mais comum entre os meses de novembro e março (DIAS et al., 1997). Todavia, de acordo com Fazolin et al. (2007) no caso da mandioca, muitas vezes a desfolha causada pelo mandarová não é suficiente para acarretar dano econômico devido à capacidade de compensação das plantas frente à falta momentânea de folhas. Plantas adultas (acima de 8 meses) são mais tolerantes, uma vez que diante de desfolhas severas podem se recuperar à custa de energia das raízes tuberosas, reduzindo a qualidade (quantidade de amido) e a produtividade.

Uma forma de estudar a ação dessa praga, visando quantificar os efeitos sobre o rendimento, é através da simulação, ou seja, provocando-se o desfolhamento artificial nas plantas. De acordo com Lima Junior et al. (2010), essa metodologia permite mensurar de maneira segura quanto de desfolha a cultura pode suportar em determinado estádio fenológico, quantificando a perda de produtividade em diferentes níveis de desfolha.

Assim, nesse trabalho objetivou-se avaliar a produtividade de plantas de mandioca quando submetida a desfolhamentos em diferentes intensidades e fases de seu desenvolvimento.

\section{METODOLOGIA}

O trabalho foi realizado na Agência Paulista de Tecnologia dos Agronegócios - Polo Regional da Alta Sorocabana, no município de Presidente Prudente, a 22ㅇ 11' de latitude S e 51을 $23^{\prime}$ de longitude W Gr. com 424,29 m de altitude. O clima é Aw, segundo classificação de Köppen, apresentando duas estações bem definidas: verão quente e úmido e inverno ameno e seco. Os dados de precipitação e temperatura média do período do experimento (junho de 2013 a maio de 2014) encontram-se na Figura 1.

Figura 1. Precipitações médias e temperaturas médias no período de junho de 2013 a maio de 2014, em Presidente Prudente/SP.

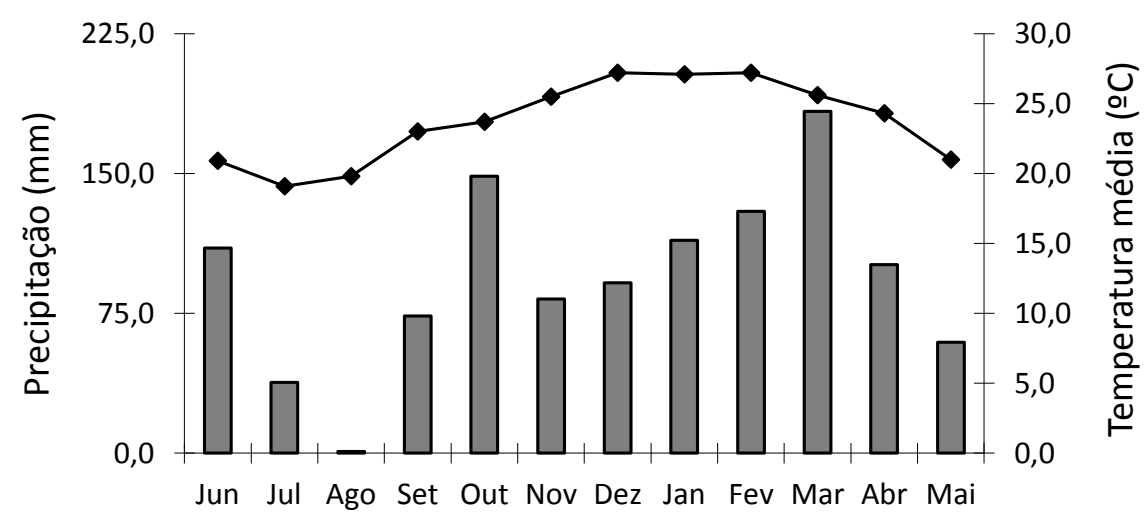

Mês

$\square$ Precipitação $\longrightarrow$ Temperatura média 
As características químicas do solo da área experimental estão descritas na Tabela 1.0 experimento foi conduzido em Argissolo Vermelho Amarelo, textura areia-franca (Tabela 2). Não foi realizada adubação de plantio ou cobertura. O delineamento experimental foi em blocos ao acaso, com quatro repetições. Os tratamentos consistiram em porcentagens de desfolha e épocas de desfolha: sem desfolha (testemunha), desfolha de 25, 50 e 75\% no mês de novembro de 2013 (120 dias após o plantio) e desfolha de 25, 50 e 75\% no mês de fevereiro de 2014 (210 dias após o plantio), perfazendo um total de 7 tratamentos.

Tabela 1 - Resultados da análise química do solo da área experimental.

\begin{tabular}{|c|c|c|c|c|c|c|c|c|c|c|c|c|c|c|c|c|c|}
\hline $\mathrm{Cu}$ & $\mathrm{Fe}$ & $\mathrm{Mn}$ & $\mathrm{Zn}$ & M.O. & $\mathrm{pH}$ & $\mathrm{P}$ & $\mathrm{K}$ & $\mathrm{Ca}$ & $\mathrm{Mg}$ & $\mathrm{Na}$ & $\mathrm{Al}$ & $\mathrm{H}+\mathrm{Al}$ & S.B. & C.T.C. & V\% & $\mathrm{s}$ & $\mathrm{B}$ \\
\hline & $\mathrm{mg}$ & $m^{-3}$ & & $\mathrm{~g} \mathrm{dm}^{-3}$ & 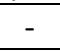 & $\mathrm{mg} \mathrm{dm}^{-3}$ & \multicolumn{8}{|c|}{$\mathrm{mmolc} \mathrm{dm}^{-3}$} & $\%$ & \multicolumn{2}{|c|}{$\mathrm{mg} \mathrm{dm}^{-3}$} \\
\hline 1,1 & 21 & 12,7 & 1,9 & 16 & 5,9 & 45 & 2,3 & 32 & 10 & - & & 14 & 44,3 & 58,3 & 76 & & 0,24 \\
\hline
\end{tabular}

Tabela 2 - Resultados da análise física do solo da área experimental.

\begin{tabular}{|c|c|c|c|c|c|}
\hline Argila (\%) & Silte (\%) & & Areia (\%) & & \\
\hline$<0,002 \mathrm{~mm}$ & $0,053-0,002 \mathrm{~mm}$ & $\begin{array}{c}\text { Total } \\
2,00-0,053 \mathrm{~mm}\end{array}$ & $\begin{array}{c}\text { Grossa } \\
2,00-0,210 \mathrm{~mm}\end{array}$ & $\begin{array}{c}\text { Fina } \\
0,210-0,053 \mathrm{~mm}\end{array}$ & $\begin{array}{c}\text { Classificação } \\
\text { textural }\end{array}$ \\
\hline 6,1 & 10,5 & 83,4 & 20,5 & 62,9 & Areia-franca \\
\hline
\end{tabular}

O plantio foi realizado em junho de 2013, quando foram utilizadas manivas com $0,2 \mathrm{~m}$ de comprimento obtidas do terço médio de hastes de plantas da cultivar de mandioca IAC 576-70, com 10 meses de idade. As manivas foram plantadas a 0,1 m de profundidade. Cada parcela apresentou quatro linhas contendo 10 plantas cada, no espaçamento de 0,7 m entre plantas e 1,0 $m$ entre linhas. A área útil constituiu-se das duas linhas centrais de cada parcela, excetuando-se as plantas das extremidades. A área experimental foi mantida sem a presença de plantas daninhas por meio de capinas manuais durante todo o ciclo da cultura da mandioca.

A desfolha foi realizada manualmente, com auxílio de tesouras de poda, sendo alternadas as folhas eliminadas e não eliminadas. Por ocasião da colheita, em maio de 2014 , foi avaliada a produção comercial por unidade de área (ha). Número de raízes total e comercial, massa fresca individual, diâmetro e comprimento de raízes comerciais também foram quantificados. Para número de raízes total foram consideradas as raízes com comprimento igual ou superior a 0,02m e $0,10 \mathrm{~m}$, respectivamente enquanto para raízes comerciais foram avaliadas as com diâmetro e comprimento igual ou superior a $0,04 \mathrm{~m}$ e $0,15 \mathrm{~m}$, respectivamente.

Os dados obtidos foram submetidos a análises de variância a $5 \%$ de probabilidade de erro.

\section{RESULTADOS}

Os níveis de desfolha e a época em que ocorreram não afetaram a produtividade que apresentou valor médio de $32 \mathrm{t} \mathrm{ha}^{-1}$ (Figura 2). 
Figura 2. Produtividade comercial de mandioca em função de épocas e níveis de desfolha.

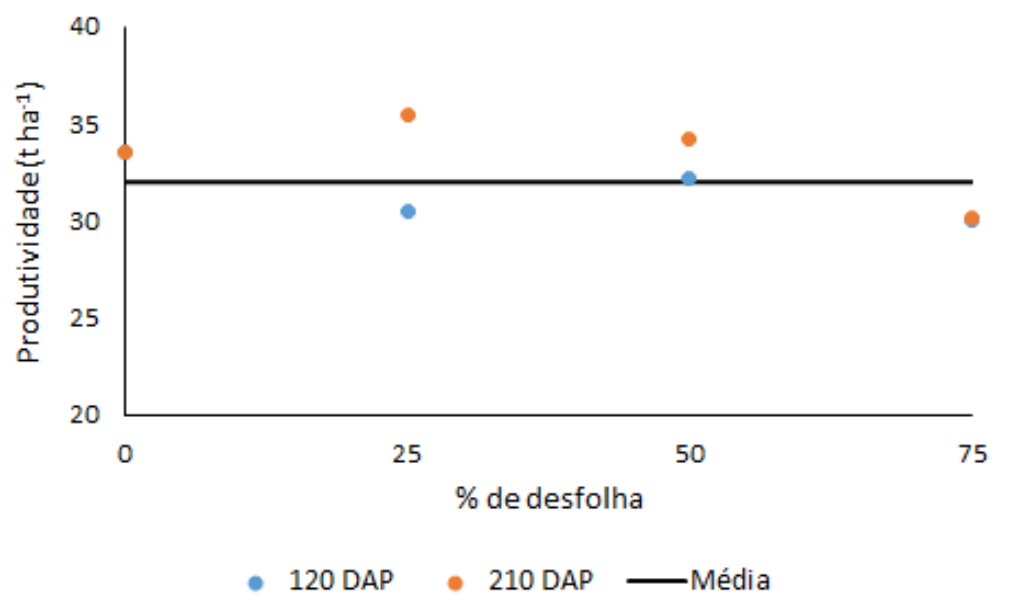

Também não foi verificada diferença estatística em números total (134.555 unidades ha ${ }^{-1}$ ) e comercial de raízes (89.930 unidades $\left.\mathrm{ha}^{-1}\right)$, massa fresca individual $(374,8 \mathrm{~g})$, diâmetro $(5 \mathrm{~cm})$ e comprimento de raízes $(26,5 \mathrm{~cm})$.

\section{DISCUSSÃO}

As desfolhas no presente trabalho ocorreram aos 120 ou 210 dias após o plantio. Como o período mais crítico para a desfolha da mandioca ocorre durante os cinco primeiros meses da cultura (LEONEL et al., 2015), esperava-se diminuição de produtividade, o que não ocorreu. Tal fato indica que a porcentagem das folhas restantes e/ou o surgimento de novas foi suficiente para manter a produtividade da cultura. De acordo com Alves (2006) o sombreamento promove uma limitação da fotossíntese o que resulta em grande parte dos fotoassimilados sendo destinados para o crescimento da parte aérea, diminuindo o crescimento das raízes de reserva, o que indica que as hastes e folhas são drenos mais fortes do que as próprias raízes, o que justifica os resultados encontrados neste trabalho. Fagundes et al. (2010) verificaram que no plantio precoce de variedades de mandioca, ocorreu elevado crescimento de ramas e dos valores de índice de área foliar máximo, o que resultou em elevada competição intra-específica e sombreamento, respectivamente, sendo verificado menor acúmulo de reservas nas raízes.

Fasae et al. (2009) também não verificaram efeito da desfolha na produtividade de mandioca em trabalho com remoção das folhas em diferentes idades das plantas. Segundo Page et al. (1980) a desfolha de plantas de mandioca somente causa perda de produtividade quando ocorre em determinadas épocas do ano e idades das plantas, pois a planta pode produzir novas folhas, compensar as perdidas e manter a produtividade.

A intensidade de perdas por desfolha em girassol (LIMA JUNIOR et al., 2010), algodoeiro (DA SILVA et al., 2012) e feijoeiro (SCHMILDT et al., 2010) também dependem do estádio fenológico das plantas. Segundo Costa et al. (2003), em soja, o sistema de preparo de solo também interfere na percentagem de perdas.

Segundo Bellotti et al. (1999), pode não haver perda na produtividade de raízes quando o solo apresenta elevada fertilidade. A área experimental do presente trabalho era ocupada por Uruchoa decumbens há cerca de 8 anos, sem qualquer exploração da área com animais. Ou seja, houve acúmulo de nutrientes em quantidade suficiente para permitir que as plantas desfolhadas apresentassem rápida recuperação.

A produtividade média de $32 \mathrm{t} \mathrm{ha}^{-1}$ encontrada neste trabalho é semelhante a reportada por Devide et al. (2009) em cultivo orgânico (33,3 $\mathrm{t} \mathrm{ha}^{-1}$ de raízes comerciais). De acordo com os 
autores, essa produtividade é considerada elevada quando comparada às lavouras comerciais do estado de São Paulo, onde a IAC 576-70 ocupa praticamente toda a área cultivada.

As condições meteorológicas do período provavelmente também foram favoráveis a recuperação das plantas que sofreram desfolha, visto que, segundo Hammer et al. (1987), no período de elevadas temperaturas as plantas de mandioca apresentam as taxas máximas de crescimento vegetativo. Ressalta-se também que as ramas apresentavam elevada sanidade e nutrição adequada, o que certamente contribuiu para que as plantas mantivessem elevada produtividade. De acordo com Takahashi (2000), a fertilidade do solo das áreas de onde são retiradas as manivas sementes influencia na produtividade das plantas originadas por essas manivas.

As justificativas que explicam a manutenção da média de produtividade, podem ser utilizadas para explicar os efeitos similares dos tratamentos nos números total e comercial de raízes, massa fresca individual, diâmetro e comprimento de raízes.

\section{CONCLUSÃO}

Nas condições edafoclimáticas em que foi realizado o experimento, a desfolha da mandioca de mesa IAC 576-70, aos 120 ou 210 dias após o plantio, não influencia a produtividade de raízes.

\section{REFERÊNCIAS}

ALLEM, A.C. The origin of Manihot esculenta Crantz (Euphorbiaceae). Genetics Resources and Crop Evolution, v.41, n.03, p.133-150, 1994. https://doi.org/10.1007/BF00051630

ALVES, A.A.C. Fisiologia da mandioca. In: EMBRAPA Mandioca e Fruticultura Tropical. Aspectos socioeconômicos e agronômicos da mandioca. Cruz das Almas, BA: EMBRAPA, 2006. Cap.7, p.138169.

BELLOTTI, A.C.; ARIAS, B.; GUZMAN, O.L. Biological control of the cassava hornworm Erinnyis ello (Lepidoptera: Sphingidae). Florida Entomologist, v.75, n.4, p.506-515, 1992. https://doi.org/10.2307/3496132

BELLOTTI, A.C.; SMITH, L.; LAPOINTE, L. S. Recent advances in cassava pest management. Annual Review of Entomology, v.44, p.343-370, 1999. https://doi.org/10.1146/annurev.ento.44.1.343

COSTA, M.A.G.; BALARDIN, R.S.; COSTA, E.C.; GRÜTZMACHER, A.D.; SILVA, M.T.B. Níveis de desfolha na fase reprodutiva da soja, cv. Ocepar 14, sobre dois sistemas de cultivo. Ciência Rural, v.33, n.5, p.813-819, 2003. http://dx.doi.org/10.1590/S0103-84782003000500004

DA SILVA, A.M.; DEGRANDE, P.E.; SUEKANE, R.; FERNANDES, M.G.; ZEVIANI, W.M.; MIGUEL, A. Impacto de diferentes níveis de desfolha artificial nos estádios fenológicos do algodoeiro. Revista de Ciências Agrárias, v.35, n.1, p. 163-172, 2012.

DEVIDE, A.C.P.; RIBEIRO, R.L.D.; VALLE, T.L.; ALMEIDA, D.L.; CASTRO, C.M.; FELTRAN, J.C. Produtividade de raízes de mandioca consorciada com milho e caupi em sistema orgânico. Bragantia, v.68, n.1, p.145-153, 2009. https://doi.org/10.1590/S0006-87052009000100016 
DIAS, C.A.C.; LONGHI, A.A.; LORENZI, J.O. Mandioca (Manihot esculenta Crantz). In: SÃO PAULO (Estado). Secretaria de Agricultura e de Abastecimento. Coordenadoria de Assistência Técnica Integral. Manual técnico das culturas. Tomo II. 2.ed. rev. atual. Campinas: CATI, 1997. p.369-398.

FAGUNDES, L.K.; STRECK, N.A.; ROSA, H.T.; WALTER, L.C.; ZANON, A.J.; LOPES, S.J. Desenvolvimento, crescimento e produtividade de mandioca em diferentes datas de plantio em região subtropical. Ciência Rural, v.40, n.12, p.2460-2466, 2010. https://doi.org/10.1590/S0103$\underline{84782010001200004}$

FAZOLIN, M.; ESTRELA, J.L.V.; CAMPOS FILHO, M.D.; SANTIAGO, A.C.C.; FROTA, F.S. Manejo integrado do mandarová-da-mandioca Erinnyis ello (L.) (Lepidoptera: Sphingidae): conceitos e experiências na região do Vale do Rio Juruá, Acre. Rio Branco, AC: Embrapa Acre, 2007. 45p. (Embrapa Acre. Documentos, 107).

HOBMAN, F.R.; HAMMER, G.L.; SHEPHERD, R.K. Effects of planting time and harvest age on cassava (Manihot esculenta) in Northern Australia. II. Crop growth and yield in a seasonally-dry environment. Experimental Agriculture, v.23, n.04, p.415-424, 1987. https://doi.org/10.1017/S0014479700017373

INSTITUTO BRASILEIRO DE GEOGRAFIA E ESTATÍSTICA - IBGE. Sistema IBGE de recuperação automática - $\quad$ SIDRA, Brasil, 2010. Disponível em: <http:// http://www2.sidra.ibge.gov.br/bda/tabela/protabl.asp?c=1612\&z=t\&o=11\&i=P>. Acesso em: 20 jun. 2017.

INTERNACIONAL POTATO CENTER. Facts and figures about sweetpotato. Lima, 2010. Disponível em: $\quad<$ http://nkxms1019hx1xmtstxk3k9sko.wpengine.netdna-cdn.com/wpcontent/uploads/PDF/005448.pdf>. Acesso em 22 jun. 2017.

LEONEL, M.; FELTRAN, J.C.; AGUIAR, E.B.; FERNANDES, A.M.; PERESSIN, V.A.; BICUDO, S.J. Mandioca (Manihot esculenta Crantz). In: LEONEL, M.; FERNANDES, A.M.; FRANCO, C.M.L. Culturas amiláceas: batata-doce, inhame, mandioca e mandioquinha-salsa. Botucatu: CERAT/UNESP, 2015. Cap.3, p.183-326.

LIMA JUNIOR, I.S.; BERONCELLO, T.F.; MELO, E.P.; DEGRANDE, P.E.; KODAMA, C. Desfolha artificial simulando danos de pragas na cultura do girassol (Helianthus annuus L., Asteraceae). Ceres, v.57, n. 1, 2015.

PAGE, W.W.; HARRIS, J.R.W.; YOUDEOWEI, A. Defoliation and consequent crop loss in cassava caused by the grasshopper Zonocerus variegatus (L.) (Orthoptera: Pyrgomorphidae) in southern Nigeria. Bulletin of Entomological Research, v.70, n.1, p.151-163, 1980. https://doi.org/10.1017/S0007485300009858

RÓS, A.B; HIRATA, A.C.S; NARITA, N. Produção de raízes de mandioca e propriedades química e física do solo em função de adubação com esterco de galinha. Pesquisa Agropecuária Tropical, v. 43, n.3, p.247-254, 2013. https://doi.org/10.1590/S1983-40632013000300001 
SCHMILDT, E. R.; AMARAL, J.A.T.; PRATISSOLI, D.; REIS, E.F. Influência de desfolhas artificiais para simular perdas na produção do feijoeiro (Phaseolus vulgaris L. cv. xamego). Arquivos do Instituto Biológico, v. 77, n.3, p. 457-463, 2010.

SCHMITT, A.T. Principais insetos pragas da mandioca e seu controle. In: CEREDA, M.P. (coord.). Agricultura: tuberosas amiláceas Latino Americanas. São Paulo: Fundação Cargill, 2002. p.350-369.

TAKAHASHI, M. Adubação com nitrogênio, fósforo e potássio na nutrição do material de propagação de mandioca e sua influência no plantio subsequente. 2000. 88f. Tese (doutorado em Agronomia) - faculdade de Ciências Agronômicas, Universidade Estadual Paulista, Botucatu. 